\title{
3D micro-printed ultra-compact single-fiber Optical tweezers
}

\author{
Innem V.A.K Reddy ${ }^{1,2}$, Andrea Bertoncini ${ }^{1}$, Carlo Liberale ${ }^{1,3}$ \\ 1. Biological and Environmental Science and Engineering Division, King Abdullah University of Science and \\ Technology, Saudi Arabia \\ 2. Department of Electrical Engineering, University at Buffalo, NY USA \\ 3. Computer, Electrical and Mathematical Science and Engineering Division, King Abdullah University of Science and \\ Technology, Saudi Arabia carlo.liberale@kaust.edu.sa
}

Abstract: We present a fully 3D printed, on-fiber hybrid photonic structure to create customizable single-fiber optical tweezers. The structure contains waveguiding, reflecting, and refracting microoptical elements stacked to generate a high-NA focal spot.

Optical tweezers (OT) are non-destructive, contactless tools that use light to trap and manipulate microscopic objects. They have applications in diverse fields, especially in biomedicine and physics [1]. Due to their unique applications and the ability to analyze sub-micron biological systems, the Nobel prize for Physics in 2018 was awarded to Arthur Ashkin, the inventor of OT. Conventional single-beam OT setups require a laser beam focusing with a high-Numerical Aperture (NA) objective. However, these setups are bulky and restrict the trapping capabilities, while the miniaturization of OT unlocks new possibilities such as trapping in turbid media or in-vivo. Over the past years, several groups attempted and successfully demonstrated fiber-based OT. Xin and their group [2] created a tapered fiber by melting and pulling the tip of a fiber. It produced a tightly focused beam out of the fiber, but with a very short working distance. To yield a high working distance, it makes sense to start with an annular/ring-shaped beam and then focus or deflect it with the help of a lens. Liu et al. $[3,4]$ illustrated the same with the help of customized annular core fiber succeeded by a ball lens or a conical optical element. Liberale et al. [5] used a bundle of four single-mode fibers along with micro-prisms, to create fiber OT with a long working distance. All the proposed approaches use non-standard fibers and grant only limited design flexibility in controlling the optical trap geometry.

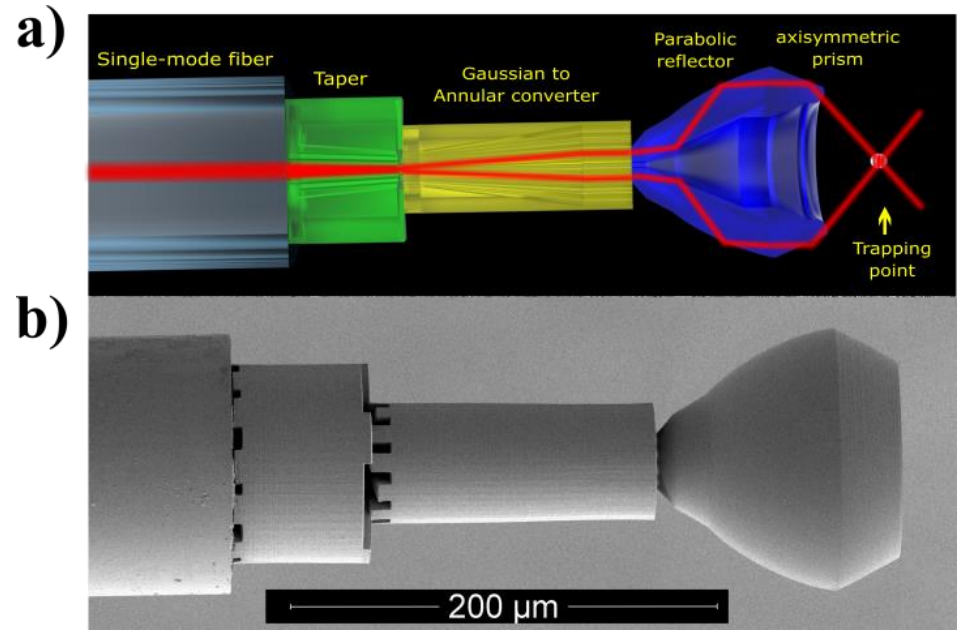

Fig. 1. (a) Design of the proposed fiber OT. It contains a taper, an gaussian to annular beam converter, a parabolic reflector and an axially symmetric prism, stacked over a single-mode fiber, (b) SEM of the fabricated structure at the tip of the fiber.

We present a novel 3D micro-printed hybrid photonic structure creating ultra-compact fiber tweezers with fully controllable optical parameters. The structure is composed of refractive, reflective, and waveguiding optical elements that are stacked together (see Fig. 1a) and is fabricated in a single step on the end-face of a standard single-mode fiber (Fig. 1b) with a commercial two-photon lithography system (Nanoscribe). The design of the different parts of the hybrid structure allows an easy and independent tuning of the trapping parameters as the 
NA and the working distance of the optical trap. In particular, a waveguide segment with photonic crystal fiber design [6] converts the Gaussian-like beam output from a standard single-mode fiber into an annular beam with a $10.5 \mu \mathrm{m}$ radius. In the second segment, the annular beam's diameter is significantly enlarged (up to $113 \mu \mathrm{m}$ ) by exploiting total internal reflection (TIR) within a suitably designed structure. The parabolic profile of one of the two reflecting surfaces allows also for the collimation of the beam. In the final segment, an axially symmetric prism-like structure deflects the annular beam by TIR to create a high-NA converging beam at the trapping point.
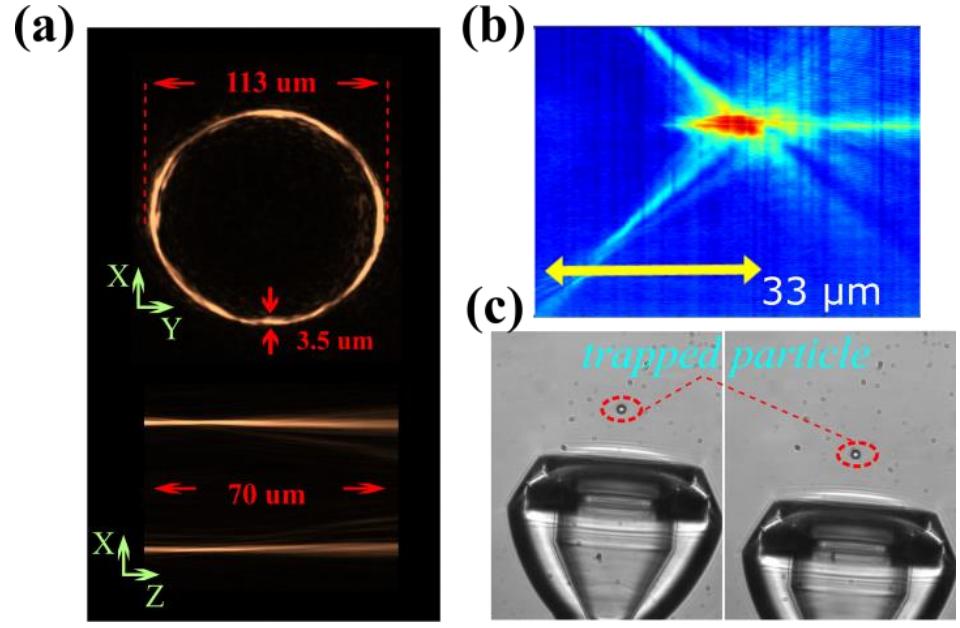

Fig. 2. (a) Annular ring output from the Parabolic reflector showcasing $X Y$ and $X Z$ plots. The figures are obtained from fabricated structure without the deflecting prism-like structure; (b) highintensity spot observed after the prism $33 \mu \mathrm{m}$ away from the structure's end; (c) a $5 \mu \mathrm{m}$ bead is trapped with the fiber OT. The parabolic reflector, prism and the bead can be seen in the figure.

One of the crucial aspects of the structure is to generate an annular beam with uniform intensity at the input of the final segment with the axially symmetric prism-like deflector. We modeled the shape and designed the parabolic reflector to yield a uniform, well-collimated annular beam (see Fig. 2a). The XY plot portrays a $113 \mu \mathrm{m}$ annular beam with a $3.5 \mu \mathrm{m}$ width, and the XZ plot indicates good collimation. The annular beam is then passed through the prism to deflect and form a high-intensity spot at a crossing point (see Fig. 2b), with sufficient optical intensity gradient to create a 3D optical trap. The working distance observed for the printed structure is around $33 \mu \mathrm{m}$. We mounted the fiber onto a cover glass for the experiment and filled the surrounding media with water along with beads ( $5 \mu \mathrm{m}$ polystyrene). We noticed a strong trap. We can see the trapped bead (Fig. 2c) away from the reflector and prism. With our design, the working distance and the NA of the fiber OT can be easily tailored by varying the diameter of the generated annular beam, and the angle of the axially-symmetric prism. A microlens can also be added to create a focused beam at the crossing point.

In conclusion, we developed a fiber OT that can be 3D printed onto a commercial single-mode fiber and is highly customizable. It is a single-step fabrication process and overcomes the limitations posed by the presentday fiber OT designs and fabrication methods.

\section{References}

[1] A. Ashkin. "History of optical trapping and manipulation of small-neutral particle, atoms, and molecules". In: leee Journal of Selected Topics in Quantum Electronics 6.6 (2000), pp. 841-856.

[2] H. B. Xin, R. Xu, and B. J. Li. “Optical trapping, driving, and arrangement of particles using a tapered fibre probe". In: Scientific Reports 2 (2012).

[3] Z. H. Liu et al. "Absorbing particle 3D trap based on annular core fiber tweezers". In: Optics Communications 437 (2019), pp. 399-402.

[4] Z. H. Liu et al. "Single fiber optical trapping of a liquid droplet and its application in microresonator". In: Optics Communications 381 (2016), pp. 371-376.

[5] C. Liberale et al. "Miniaturized all-fibre probe for three-dimensional optical trapping and manipulation". In: Nature Photonics 1.12 (2007), pp. 723-727.

[6] A. Bertoncini and C. Liberale. "3D printed waveguides based on photonic crystal fiber designs for complex fiber-end photonic devices". In: Optica 7.11 (2020), pp. 1487-1494. 